\title{
Preferential effect of synchrotron microbeam radiation therapy on intracerebral 9L gliosarcoma vascular networks
}

Audrey Bouchet, $\mathrm{MS}^{*}$,, Benjamin Lemasson, $\mathrm{MS}^{\dagger, \dagger \dagger, \$,}$, Géraldine Le Duc, $\mathrm{PhD}^{*}$, Cécile Maisin, MS ${ }^{\dagger, \dagger \dagger}$, Elke Bräuer-Krisch, MS ${ }^{*}$, Erik A. Siegbahn, PhD" ${ }^{\|}$Luc Renaud ${ }^{\mathbb{I}, \#}$, Enam Khalil, $\mathrm{PhD}^{\ddagger}$, Chantal Rémy, $\mathrm{PhD}^{\dagger, \dagger \dagger}$, Cathy Poillot, $\mathrm{MS}^{\dagger, \dagger \dagger}$, Alberto Bravin, $\mathrm{PhD}^{*}$, Jean A. Laissue, $\mathrm{MD}^{\$}$, Emmanuel L. Barbier, $\mathrm{PhD}^{\dagger, \dagger \dagger}$ and Raphaël Serduc, $\mathrm{PhD}^{*}$

${ }^{*}$ ESRF, Grenoble, France

${ }^{\dagger}$ INSERM, U836, Grenoble, F-38043, France

${ }^{\dagger \dagger}$ Université Joseph Fourier, Grenoble Institut des Neurosciences, UMR-S836, Grenoble, F38043, France

${ }^{\S}$ Oncodesign Biotechnology, Dijon, France

"Department of Medical Physics, Karolinska Hospital, Stockholm, Sweden

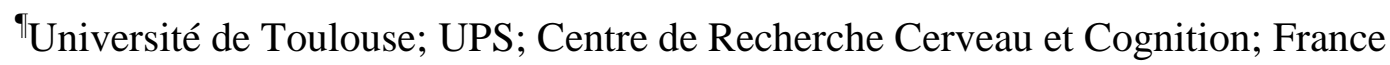

${ }^{\#}$ CNRS; CerCo; Toulouse, France

${ }^{\ddagger}$ Faculty of Pharmacy, University of Jordan, Amman, Jordan

${ }^{\$}$ Institute of Pathology, University of Bern, Switzerland

Equally contributed to the work.

Reprint requests: Raphaël Serduc. Monash Institute of Medical Research, 3168, ClaytonVictoria. Australia. E-mail: raph.serduc@gmail.com

Running title: MRT preferentially damages tumor vasculature

Conflict of interest notification: none. 


\section{Abstract}

Purpose. Synchrotron Microbeam Radiation Therapy (MRT) relies on spatial fractionation of the incident photon beam into parallel micron-wide beams. Our aim was to analyze the effects of MRT on normal brain and 9L gliosarcoma tissues particularly on blood vessels.

Methods and Materials. Responses to MRT (2 arrays, one lateral, one antero-posterior (2x400Gy), intersecting orthogonally in the tumor region), were studied during 6 weeks using MRI, immunohistochemistry and VEGF western blot.

Results. MRT increased the median survival time of irradiated rats (x3.25), significantly increased blood vessel permeability and inhibited tumor growth; a cytotoxic effect on 9L cells was detected 5 days after irradiation. Significant decreases in tumoral blood volume fraction and vessel diameter were measured from day 8 after irradiation, due to loss of endothelial cells in tumors as detected by immunochemistry. An edema was observed in the normal brain exposed to both crossfired arrays about 6 weeks after irradiation. This edema was associated with changes in blood vessel morphology and an overexpression of VEGF. Conversely, vascular parameters and vessel morphology in brain regions exposed to one of the two arrays were not damaged, without loss of vascular endothelia.

Conclusions. We show for the first time that preferential damage of MRT to tumor vessels versus preservation of radioresistant normal brain vessels contributes to the efficient palliation of 9L gliosarcomas in rats. Molecular pathways of repair mechanisms in normal and tumoral vascular networks after MRT may be essential for the improvement of such differential effects on the vasculature.

Key words: Synchrotron Microbeam radiation therapy/intracerebral 9L gliosarcoma/tumor vasculature 


\section{Purpose}

Tumor vasculature is deemed to be a promising target for the treatment of solid cancerous lesions. Different therapeutic approaches, alone or combined, such as antiangiogenic therapy, chemo-, radio- and immunotherapy are currently refined in order to specifically interfere with blood vessel growth in the tumor (1). This objective might be also achieved by a novel form of radiosurgery, known as Microbeam Radiation Therapy (MRT) (2), which uses a particular irradiation geometry and X-rays generated in a synchrotron light source; the beam is spatially fractionated into an array of quasi-parallel planar microbeams which are a few tens microns wide and separated by a few hundred microns. This unique irradiation geometry allows very high dose (hectograys) deposition efficient for the treatment of brain tumors in small animals while ensuring an unusually high normal tissue tolerance, even in developing larger animals $(3,4)$.

The preferential effect on malignant tissues has been mainly attributed to selective effects of microbeams on immature tumor vessels versus lack of microbeam effects on the differentiated normal vasculature (5), although other factors might be operative. Lesions induced by the microbeams in the normal capillary network can be rapidly repaired $(6,7)$ and normal arteries are microbeam-resistant too (8). Thus, there is no decrease in vessel density, blood volume, no hemorrhage and no ischemic necrosis after MRT in normal mammalian tissues. Conversely, no clear experimental proof of the effect of MRT on tumor vessels has been given in the literature. Our recent magnetic resonance imaging study failed to reveal important damage to tumor vessels after MRT (9). Crossfired MRT slowed the growth of the heterologously implanted 9L gliosarcoma (9LGS) and the development of its vasculature. We now characterize the biological effects of $50 \mu \mathrm{m}$-wide microbeams, efficient in terms of tumor control and normal tissue sparing (10), on normal rat brain and intracerebral 9LGS, 
Bouchet et al. 2010

particularly on growth and morphometric parameters of blood vessels from day 2 to day 45 after irradiation, and on the expression of the Vascular Endothelial Growth Factor. 


\section{Methods and Materials}

All procedures related to animal care conformed to the Guidelines of the French Government with licenses 380325 and 380321 . Rats were anesthetized with an intraperitoneal injection of xylazine/ketamine $\left(64.5 / 5.4 \mathrm{mg} \mathrm{kg}^{-1}\right.$ for irradiations) and $2.5 \%$ isoflurane (MRI follow-up) for maintenance.

\section{Tumor inoculation}

Transplantable 9LGS cells were implanted in 59, 10-week-old-Fisher rats weighing between 180 and 220g. Implantation procedure followed the protocol described in (10). Briefly, $10^{4}$ 9LGS cells suspended in a $1 \mu$ DMEM medium were injected into the right caudate nucleus $(3.5 \mathrm{~mm}$ from the bregma) at a depth of $5.5 \mathrm{~mm}$ from the dura.

\section{Magnetic resonance imaging and group sorting}

We used a 4.7T Bruker Avance console and volume/surface cross coil configuration. Seven days after 9LGS implantation, rats underwent anatomical MRI ( $\mathrm{T}_{2}$-weighted image) in order to sort them two groups with similar mean tumor size. Those 59 rats were divided into a control group $(\mathrm{n}=25)$ and a group of 34 rats and irradiated by MRT 10 days after 9LGS implantation. Rats ( $\mathrm{n}=4$ to 8 ) underwent MRI and 4 of them were culled at each delay for biological studies. The survival curves were established using 9 rats of each group. The number of days elapsed after implantation (n) and treatment $(m)$ is designated as $D_{n} T_{m}$.

\section{Radiation source and irradiation geometry}

Irradiations were performed on the ID17 biomedical beamline at the European Synchrotron Radiation Facility using X-rays emitted tangentially from relativistic electron bunches circulating in a storage ring. The wiggler produces a wide spectrum of photons which extends 
from 50 over $350 \mathrm{keV}$ (median energy: $90 \mathrm{keV}$ ). The quasi-laminar beam was shaped into an array (8mm wide) of rectangular, microscopically thin and quasi-parallel microbeams using a multislit collimator (11). Rats in the treated group were irradiated $\left(\mathrm{D}_{10} \mathrm{~T}_{0}\right)$ after tumor inoculation using two, $10 \mathrm{~mm}$ high, arrays of 40 vertical, planar microbeams (width $50 \mu \mathrm{m}$, $200 \mu \mathrm{m}$ on-center spacing), one anteroposterior, the other lateral, both focused for orthogonal intersection (crossfiring) in the anterior part of the right hemisphere, where the 9LGS was located. Before reaching and after leaving the volume of intersection enclosing the tumor, each array alone covered a volume of the rat's head in which it was unidirectional. Animals were aligned into the beam using 3 live cameras and the coordinates of the center of the microbeam arrays were $3.5 \mathrm{~mm}$ left from the medio-lateral line, $-6 \mathrm{~mm}$ in the dorso-ventral direction from the skin surface and at the bregma point in the anterioposterior direction. The in-microbeam entrance dose was 400Gy per array, the mean dose rate $\sim 16000 \mathrm{~Gy} \cdot \mathrm{s}^{-1}$.

\section{Monte Carlo simulation and doses calculations}

The doses were calculated by means of the Monte Carlo method normally used for MRT dose planning and quality assurance at the ESRF (12). Further details of our MRT dosimetry protocols are provided as Supplementary Material.

\section{Rat follow-up by MRI}

Eight rats were imaged one day before $\left(\mathrm{D}_{9} \mathrm{~T}_{-1}\right)$ and animals from all groups at 2, 5,8 15 and 45 days after MRT treatment. For each group, 4 rats were followed up and imaged at every time point. Four rats per groups were also imaged at every delay after irradiation and culled for immunohistological studies and western blot analysis.

Each MRI session (duration 1h15), detailed in the Supplementary material, was organized as follow: 
- Anatomical $\mathrm{T}_{2}$-weighted images

- BVf (blood volume fraction) and VSI (vessel size index) measurements

- Vascular permeability of P846 (Gd-based contrast agent) measurements

\section{MRI data analysis}

BVf, VSI and vessel permeability maps were computed pixel by pixel using an inhouse program developed under Matlab (MathWorks, NA). BVf and VSI were calculated as described previously $(13,14)$. Null pixels were excluded in the mean calculation in order to give information only on perfused brain regions. Vessel permeability was estimated by subtracting the peak MR signal obtained after the injection of Gd-DOTA from the peak MR signal measured before injection and expressed as a percentage of contrast enhancement. Three regions of interest (ROIs) were manually delineated on the $\mathrm{T}_{2}$-weighted image on which the tumor appeared the largest (see fig. 1) i) the whole tumor, ii) the ipsilateral (right) hemisphere without tumor and iii) the contralateral hemisphere, and reported on the other maps. Tumor and surrounding parts of the normal brain in the right hemisphere were exposed to the two intersecting arrays, normal brain parts of the left hemisphere unidirectionally to the lateral array alone. Tumor volumes were determined by multiplying the pixel size by the number of "tumoral pixels" on all MR slices on which the tumor was visible. Two-way ANOVA followed by Bonferroni post-hoc tests were used for statistics. A Mann-Whitney test was used to compare 9LGS volumes. The statistical analyses were performed with the Prism program (GraphPad Software, USA). Values were considered significantly different when $\mathrm{p}<0.05$.

\section{Immuno / histological analyses (IHC)}

Type-IV collagen $\left(\mathrm{C}_{\mathrm{IV}}\right)$, a protein constituent of the vessel basal lamina, and RECA-1 (Rat Endothelial Cell Antigen), an antigen of endothelial cells, were labeled as previously 
described $(7,15)$. Cell proliferation activity was assessed by means of Ki67 labeling. Further details of our IHC protocols and quantification are provided as Supplementary Material.

\section{Western blot analysis of VEGF}

The western blot protocol of VEGF is described in the Supplementary Material section. 


\section{Results}

The main results of this study are summarized in table 1. Radiation doses were calculated in a rat head phantom using Monte Carlo simulations. The peak dose deposited at $1 \mathrm{~cm}$ depth in rat brain was 350/700Gy, generating a valley dose of $12.5 / 25 \mathrm{~Gy}$ in the unidirectionally/bidirectionally irradiated brain regions respectively.

\section{Survival curves}

Figure 1C shows the Kaplan-Meier representation of the survival of untreated and MRT-treated 9LGS bearing rats. MRT significantly increased the MST (mean survival time) of treated animals compared with the control group of non-irradiated rats (65 days versus 20 , respectively, log rank test: $\mathrm{p}=0.003$ ).

\section{MRT effects on 9LGS growth}

Figure 1A shows a $\mathrm{T}_{2}$-weighted MRI follow-up of irradiated and non irradiated 9LGS which grew exponentially and equally between $\mathrm{D}_{9} \mathrm{~T}_{-1}$ and $\mathrm{D}_{18} \mathrm{~T}_{8}$ and reached comparable sizes at $\mathrm{D}_{12} \mathrm{~T}_{2}\left(15.2 \pm 5.3 \mathrm{~mm}^{3}\right.$ in non irradiated versus $7.0 \pm 4.8 \mathrm{~mm}^{3}$ in irradiated rats). Tumor volume measured on $\mathrm{T}_{2}$-weighted MR images are reported in figure $1 \mathrm{~B}$. The effects of MRT were observed from $\mathrm{D}_{15} \mathrm{~T}_{5}$ : tumors were significantly smaller in MRT-treated rats compared with controls, by a factor of about 17 at $\mathrm{D}_{18} \mathrm{~T}_{8}$ (fig. $13.9 \pm 9.7 \mathrm{~mm}^{2}$ (MRT) versus $231.7 \pm 51.3 \mathrm{~mm}^{3}$ (untreated), $\mathrm{p}<0.001$ ). Later, between $\mathrm{D}_{9} \mathrm{~T}_{-1}$ and $\mathrm{D}_{25} \mathrm{~T}_{15}$, no difference in tumor size was found. Thereafter, the evaluation of tumor volume using on $\mathrm{T}_{2}$-weighted MR images became more problematic since 2 rats did not exhibit tumor anymore (the hyposignal as shown in figure 1Ah was taken into account for lesion size determination); 2 rats showed small tumors and 1 rat a recurrent tumor comparable to those measured in control rats at $D_{18} T_{8}$. 


\section{MRT effects on 9LGS proliferative activity}

The analyses Ki67 immunolabeling and HE stained sections revealed that MRTinduced damages were detectable from $\mathrm{D}_{15} \mathrm{~T}_{5}$ (fig. 2): cell density decreased on H\&E stained brain sections in irradiated tumors; the proliferative activity (proportion of Ki67 positive cells) of the tumor was distinctly reduced. Giant tumoral cells were observed. At $\mathrm{D}_{25} \mathrm{~T}_{15}$, the 9LGS tissue was strongly disorganized, exhibiting cellular polymorphism and large lacunae and/or necrotic regions in the core. Only few dividing cells were found at the tumor periphery. For two thirds of the animals observed at $\mathrm{D}_{55} \mathrm{~T}_{45}$ by immunohistology, it was not possible to delimit a tumor. Conversely, one third of the animals presented a tumor with numerous Ki67 positive cells which had invaded the whole ipsilateral hemisphere (fig. 2). Normal tissue exhibited astrocyte and neuron loss in the microbeam paths starting at day 5 after irradiation.

\section{MRT effects on the evolution of normal and tumoral blood vessels of the brain Blood volume fraction}

Cerebral BVf (fig. 3) were significantly higher in the 9LGS than in the ipsi- and contralateral normal brain for all observation times. A decrease in tumoral blood volume fraction (TBVf) was detected between $\mathrm{D}_{9} \mathrm{~T}_{-1}$ and $\mathrm{D}_{18} \mathrm{~T}_{8}$ in irradiated or non-irradiated tumors ( $\mathrm{p}<0.001)$, whereas the CBVfs of the ipsi- and contralateral normal hemisphere did not change. Thereafter, in irradiated animals, TBVf decreased between $D_{18} T_{8}$ and $D_{25} T_{15}$ $(<0.001)$

\section{Vessel size index}

VSI values increased similarly in both irradiated and non irradiated tumors until D18, where they were significantly higher in tumors than in normal tissues $(\mathrm{p}<0.001)$. Thereafter, 
VSI values decreased in irradiated tumors between $\mathrm{D}_{18} \mathrm{~T}_{8}$ and $\mathrm{D}_{25} \mathrm{~T}_{15}(-23.8 \%, \mathrm{p}<0.001)$. In normal tissues surrounding the tumor within the volume targeted by the intersecting, crossfired arrays (ipsilateral+MRT), VSI increased between $\mathrm{D}_{18} \mathrm{~T}_{8}$ and $\mathrm{D}_{55} \mathrm{~T}_{45}$ (8.5 to $13.7 \mu \mathrm{m}$, $\mathrm{p}<0.001)$

\section{Contrast enhancement}

Vessel permeability (indicated as contrast MR enhancement, figure 3) was significantly higher in 9LGS than in the normal ipsi- or contralateral hemisphere, and it was not modified in normal brain tissue after unidirectional or crossfired irradiations. Conversely, MRT led to an increase in tumor vessel permeability during the 5 days following the irradiation: The MR signal enhancement reached $26.6 \%$ at D15 while it was only $20.3 \%$ in nonirradiated tumors $(\mathrm{p}<0.001)$. Then, the blood vessel permeability of irradiated tumors decreased by $74 \%$ between $\mathrm{D}_{15} \mathrm{~T}_{5}$ and $\mathrm{D}_{25} \mathrm{~T}_{15}(\mathrm{p}<0.001)$.

\section{Blood vessels; immunohistological observations}

Vascular networks were observed on brain sections labeled by $\mathrm{C}_{\mathrm{IV}}$ (basal membrane) and RECA-1 (endothelial cells, fig. 4-5). The co-expression ratio of these proteins in vessels is reported in on each image on Figure 4. During the entire experiment, no damage was detected in the unidirectionally irradiated volume of the contralateral hemisphere (fig. 4). All brain capillaries were double stained by $\mathrm{C}_{\mathrm{IV}}$ / RECA-1 antisera. Conversely, the morphology of blood vessels located in the normal brain tissue surrounding the tumor within the volume of intersecting arrays changed around $\mathrm{D}_{55} \mathrm{~T}_{45}$ : the normal vascular network showed an important disorganization and increased tortuosity. $\mathrm{C}_{\mathrm{IV}}$ immunolabeling revealed the presence of narrow (diameter 1-2 $\mu \mathrm{m}$ ) entities without endothelial cells, as shown by a quasi-complete lack of 
reactivity for RECA-1 labeling. Few capillaries of normal size lacked homogenous RECA-1 labeling.

Tumor vessel density was lower than that observed in normal brain tissue. Spatial vessel distribution was irregular and vessel diameters were distinctly larger compared with normal brain blood vessels. The number of blood vessels per surface area in non-irradiated as well as in irradiated tumors decreased with time after implantation. However, beginning at $\mathrm{D}_{12} \mathrm{~T}_{2}$, RECA-1 labeling intensity gradually faded in 9LGS vessels in the beam-crossing volume. In tumors observed from $\mathrm{D}_{18} \mathrm{~T}_{8}$ to $\mathrm{D}_{55} \mathrm{~T}_{45}$, most of 9LGS vessels were not immunoreactive for RECA-1, as shown in figure 5 for a rat culled at $\mathrm{D}_{18} \mathrm{~T}_{8}$.

\section{MRT effects on VEGF expression}

No VEGF expression was detected in contralateral (left) hemispheres of control rats during the whole experiment. Conversely, MRT induced an expression of VEGF increasing with time in normal brain tissues, i.e., from $\mathrm{D}_{12} \mathrm{~T}_{2}$ or $\mathrm{D}_{15} \mathrm{~T}_{5}$, in both hemispheres, whether irradiated by one or by both intersecting arrays (Fig. 6). At $\mathrm{D}_{55} \mathrm{~T}_{45}$, the amount of VEGF detected in normal brain parts of the ipsilateral hemisphere situated in the beam-crossing region was $>2.5$-times higher $(\mathrm{p}<0.001)$ than the one measured in the left hemisphere irradiated unidirectionally by the lateral array (Contra+MRT).

In 9LGS, the relatively low expression of VEGF did not markedly change during their growth (fig. 6). Conversely, the irradiation led to a progressive and significant increase in VEGF expression in tumors. Indeed, the ratio VEGF/GADPH changed from 0.4 to 1.1 between $D_{12} T_{2}$ and $D_{18} T_{8}$ while it fluctuated from 0.5 to 0.8 in untreated tumors during the same period (NS for untreated tumors, $\mathrm{p}<0.05$ in irradiated tumors). At $\mathrm{D}_{25} \mathrm{~T}_{15}$ and $\mathrm{D}_{55} \mathrm{~T}_{45}$, the 
Bouchet et al. 2010

relative expression of VEGF increased significantly in irradiated tumors $(\mathrm{p}<0.001)$, to values 1.35 and 1.8 times higher than the one measured at $\mathrm{D}_{18} \mathrm{~T}_{8}$. 


\section{Discussion}

The crossfired irradiation increased significantly the MST of rats bearing intracerebral 9LGS compared with unirradiated controls $(\mathrm{p}=0.0003)$. MRT inhibited tumor growth during few weeks after treatment as shown by MRI. Histopathologically, MRT induced a decrease in tumor cell density and reduced the proliferative activity of the lesion. The salient findings in this study were 1) the preferential loss of endothelial cells in tumor vessels exposed to crossfired arrays, from day 5 after treatment and 2) the significant decrease in tumoral blood volume fraction during the second week after irradiation; conversely, the microvasculature and the perfusion in the normal brain volumes irradiated unidirectionally in the contralateral hemisphere remained undamaged. Normal blood vessels in peritumoral normal brain tissue were damaged by both intersecting arrays.

The hypothesis of a preferential effect of MRT on tumoral blood vessels rather than on normal vasculature is well established $(5,16)$. However, Serduc et al. described a limited influence of the vascular component during 9LGS palliation in mice by MRT (9). A significant increase in tumor vessel permeability on $\mathrm{D}_{12} \mathrm{~T}_{2}$ and $\mathrm{D}_{15} \mathrm{~T}_{5}(+44 \%$ and $+23 \%)$ preceded tumor necrosis in the present experiment. No change in blood-brain barrier permeability was detected in ipsilateral (exposed to both arrays) and contralateral (exposed to one array only) normal brain tissues. This transient tumor-blood barrier might be used to deliver intravenously injected drugs to the tumor core. Adjuvant chemotherapy would be justified by the fact that in previous studies MRT did not sterilize intracerebral 9LGS, a highly resistant gliosarcoma model, even when adequate irradiation configurations and valley doses were used (45Gy (15), 36Gy (17)). In the present experiment, tumor growth was inhibited during several weeks after MRT in association with reduced proportions of proliferative Ki67-positive tumor cells, starting on $\mathrm{D}_{15} \mathrm{~T}_{5}$. Two weeks after MRT, large 
necrotic regions were detected in the tumor core. The presence of proliferative cells at the tumor periphery may indicate tumor recurrence.

As several rats survived for a very long period after MRT for 9LGS in other experiments $(5,16)$, despite the presence of $\sim 2 \%$ surviving clonogenic 9LGS cells in the tumor (5), it was suggested that tumor ablation was due to the preferential effect of MRT on tumor blood vessels resulting in ischemic necrosis of the tumor. However, in the present study, morphometric parameters of the vascular network were not affected shortly after MRT, i.e. between $\mathrm{D}_{12} \mathrm{~T}_{2}$ and $\mathrm{D}_{18} \mathrm{~T}_{8}$. Indeed, we observed no difference in $\mathrm{BVf}$ and VSI values between irradiated and non irradiated tumors. Conversely, during this period, RECA-1 labeled endothelia were not detected in all Type-IV collagen positive vascular structures of irradiated tumors, while their vascular counterparts in the time-matched non-irradiated tumors exhibited obvious double staining. Loss of RECA-1 immunoreactivity indicates a loss of endothelial cells. Alternatively, some vessels might have been occluded by thrombosis or following endothelial denudation. However, BVf and VSI values measured by MRI after Sinerem iv. injection did not indicate a decreased tumor perfusion after irradiation. Thus, the loss of endothelial cells might not have measurably impaired tumor blood supply after MRT. Loops of extracellular matrix, devoid of endothelia, seem able to temporarily irrigate tumors with blood (18). Such $\mathrm{C}_{\mathrm{IV}}$ positive vessel-like structures lacking immunoreactive endotheliocytes, might thus permit a transitory perfusion of 9GLS; the parameters showing the evolving perfusion after irradiation in the same animal support this interpretation (Fig. 1A): after $\mathrm{D}_{18} \mathrm{~T}_{8}$, tumor size reduction until $\mathrm{D}_{25} \mathrm{~T}_{15}$ correlates with the significant decrease in TBVf and in VSI (respectively $-24.6 \%, \mathrm{p}<0.05$ and $-23.8 \%, \mathrm{p}<0.01$ ). Conversely, BVf and VSI values, and morphology of brain vessels in the normal brain parts exposed to crossfired arrays remained unchanged during the same period. These data persuasively support the existence of a 
preferential damaging effect of MRT on tumor vessels. During the second week after treatment, tumor necrosis can be associated with the delayed decrease in TBVf and VSI.

The use of $50 \mu \mathrm{m}$-wide microbeams significantly improved the MSTs for rats with 9LGS in our laboratory $(15,17)$, notably in this study. This particularly radioresistant tumor requires very high radiation dose deposition for palliation or even ablation; a 40Gy radiosurgical dose controlled only $50 \%$ of 9 LGS (19). Tumor responses to MRT on $\mathrm{D}_{55} \mathrm{~T}_{45}$ in our study were markedly heterogeneous Indeed, 2/5 rats did not exhibit tumors; 2 animals showed well-delimited small tumors and 1 rat had a large tumor invading the whole hemisphere. The cause for these different responses is not understood but these 5 rats displayed a common change in MRI, a cerebral edema in parts of the ipsilateral hemisphere exposed to both intersecting arrays. These results were supported by demonstration of infiltrates of monocytes/macrophages (Supplementary materials, Fig. e1) and of a strong disorganization and remodeling of the normal vascular network at this site. Normal vessels appeared tortuous and larger, an observation consistent with the significant increase in VSI at $\mathrm{D}_{55} \mathrm{~T}_{45}(+9.8 \%)$. Thin matricial entities, probably not perfused, were commonly observed. RECA-1 labeling was inhomogeneous.

A gradual increase in VEGF expression was detected in tumoral and in normal tissues. However, the kinetics and the amount of the protein produced seemed to depend on the radiation dose (exposure to uni- or bidirectional irradiation) and the status of the tissue (normal or tumoral). VEGF, secreted e.g. in ischemia, stroke and after irradiation $(20,21)$, is commonly induced in presence of hypoxia; changes in tumoral and normal brain oxygen saturation after MRT should thus be evaluated. VEGF in the ipsilateral hemisphere was detectable from the second day after MRT and reached levels comparable to those measured in non-irradiated tumors. Thus, VEGF is implied 
in cerebral response in the early phase after MRT but its role is not defined. The overexpression of VEGF after MRT could have increased blood vessel permeability and led to severe brain edema. Since the size of the tumors did not change significantly between $D_{25} T_{15}$ and $D_{55} T_{45}$, we assume that MRT-induced brain edema in the crossfired regions was the main cause of animal deaths in our study. These qualitative findings are consistent observations (16) that major histopathologic brain and vascular damage was confined to MRT beam-crossing regions, whereas only minor damage was apparent in zones of the brain irradiated unidirectionally (16). A comprehensive and detailed quantitative brain damage index for the present study was not established. However, the histopathologic findings were comparable, despite the different irradiation modalities.

Cross-fired MRT , particularly efficient for tumor control, may require corticosteroid injections (22) to control ensuing brain edema. Further, recovery from radiation injury may be influenced by growth factors involved in cell renewal and differentiation of neural tissue, e.g. by VEGF. Thus, a brief therapy with VEGF can prevent or delay radiation myelopathy after spinal cord injury in rats (23). Experimentally, inhibition of VEGF may increase local tumor control after irradiation, possibly by preventing tumor angiogenesis (21).

The preferential effect of MRT on tumor vasculature may lead to further improvements. Most importantly, the tumor targeting for MRT used preclinically must be improved and adapted to clinical standards. Irradiation fields must be limited to the tumor; image guided, non coplanar irradiations, which limit normal tissue damage, should be adopted $(15,24)$. 


\begin{tabular}{|c|c|c|c|}
\hline Parameter & $\begin{array}{c}\text { Blood } \\
\text { vessels in }\end{array}$ & Irradiation & Observations \\
\hline \multirow{3}{*}{$\begin{array}{l}\text { Blood vessel } \\
\text { morphology }\end{array}$} & \multirow{2}{*}{$\begin{array}{l}\text { Normal } \\
\text { Brain }\end{array}$} & $\begin{array}{l}\text { UNIDIRECTIONAL } \\
\text { (UNI) }\end{array}$ & \multirow{3}{*}{$\begin{array}{l}\text { No changes. Endothelial cells and } \\
\text { vascular basement membranes are } \\
\text { immunolabeled } \\
\text { Long term changes }\left(\mathrm{D}_{55} \mathrm{~T}_{45}\right) \text { Increased } \\
\text { tortuosity. Narrow channels without } \\
\text { endothelia. Reduced RECA reactivity. } \\
\text { Short Term changes }\left(\mathrm{D}_{12} \mathrm{~T}_{2}\right) \text {. Gradual } \\
\text { decrease of RECA reactivity. } \\
\text { Loss of tumor endothelia } \\
\text { (denudation) }\end{array}$} \\
\hline & & $\begin{array}{l}\text { CROSSFIRED } \\
\text { (CROSS) }\end{array}$ & \\
\hline & Tumor & CROSS & \\
\hline \multirow{3}{*}{$\begin{array}{l}\text { Blood Volume } \\
\text { Fraction (BVf) }\end{array}$} & \multirow{2}{*}{$\begin{array}{l}\text { Normal } \\
\text { Brain }\end{array}$} & UNI & No changes \\
\hline & & CROSS & No changes \\
\hline & Tumor & CROSS & Significant decrease from $\mathrm{D}_{18} \mathrm{~T}_{8}$ \\
\hline \multirow{3}{*}{$\begin{array}{l}\text { Vessel Size } \\
\text { Index (VSI) }\end{array}$} & \multirow{2}{*}{$\begin{array}{l}\text { Normal } \\
\text { Brain }\end{array}$} & UNI & No changes \\
\hline & & CROSS & Significant increase at $\mathrm{D}_{55} \mathrm{~T}_{45}$ \\
\hline & Tumor & CROSS & Significant decrease from $\mathrm{D}_{18} \mathrm{~T}_{8}$ \\
\hline \multirow{3}{*}{$\begin{array}{c}\text { Vessel } \\
\text { Permeability }\end{array}$} & \multirow{2}{*}{$\begin{array}{l}\text { Normal } \\
\text { Brain }\end{array}$} & UNI & No changes \\
\hline & & CROSS & \multirow{2}{*}{$\begin{array}{c}\text { No changes } \\
\text { Short term increase at } \mathrm{D}_{12} \mathrm{~T}_{2} \text { and } \\
\mathrm{D}_{15} \mathrm{~T}_{5}\end{array}$} \\
\hline & Tumor & CROSS & \\
\hline
\end{tabular}

Table 1: MRT effects on blood vessels located either in a 9LGS tumor or in normal brain tissue. Parts of left hemisphere were irradiated unidirectionally by the lateral array (UNI). Extratumoral anterior parts of the right hemisphere were irradiated by the intersecting lateral and anteroposterior arrays (CROSS). The 9LGS tumor in the right anterior hemisphere was also cross-irradiated. The number of days (n) elapsed after implantation or irradiation is designated as DnTn, respectively. RECA $=$ Rat Endothelial Cell Antigen. 


\section{References}

1. Jain RK, di Tomaso E, Duda DG, et al. Angiogenesis in brain tumours. Nat Rev Neurosci 2007;8:610-622.

2. Slatkin DN, Spanne P, Dilmanian FA, et al. Subacute neuropathological effects of microplanar beams of x-rays from a synchrotron wiggler. Proc Natl Acad Sci U S A 1995;92:8783-8787.

3. Brauer-Krisch E, Serduc R, Siegbahn EA, et al. Effects of pulsed, spatially fractionated, microscopic synchrotron X-ray beams on normal and tumoral brain tissue. Mutat Res 2009.

4. Laissue JA, Blattmann H, Wagner HP, et al. Prospects for microbeam radiation therapy of brain tumours in children to reduce neurological sequelae. Dev Med Child Neurol 2007;49:577-581.

5. Dilmanian FA, Button TM, Le Duc G, et al. Response of rat intracranial 9L gliosarcoma to microbeam radiation therapy. Neuro-oncol 2002;4:26-38.

6. Serduc R, van de Looij Y, Francony G, et al. Characterization and quantification of cerebral edema induced by synchrotron x-ray microbeam radiation therapy. Phys Med Biol 2008;53:1153-1166.

7. Serduc R, Verant P, Vial JC, et al. In vivo two-photon microscopy study of short-term effects of microbeam irradiation on normal mouse brain microvasculature. Int J Radiat Oncol Biol Phys 2006;64:1519-1527.

8. Van der Sanden B, Bräuer-Krisch E, Siegbahn EA, et al. Tolerance of arteries to microplanar X-ray beams. In print Int J Radiat Oncol Biol Phys 2010.

9. Serduc R, Christen T, Laissue J, et al. Brain tumor vessel response to synchrotron microbeam radiation therapy: a short-term in vivo study. Phys Med Biol 2008;53:3609-3622.

10. Serduc R, Bouchet A, Brauer-Krisch E, et al. Synchrotron microbeam radiation therapy for rat brain tumor palliation-influence of the microbeam width at constant valley dose. Phys Med Biol 2009;54:6711-6724.

11. Bräuer-Krisch E, Requardt H, Brochard T, et al. New technology enables precision multi slits collimators for MRT (Microbeam Radiation Therapy). Accepetd Review of Scientific Instruments 2009.

12. Siegbahn E, A., Stepanek J, Bräuer-Krisch E, et al. Determination of dosimetrical quantities used in microbeam radiation therapy (MRT) with Monte Carlo simulations. Med. Phys. 2006;33:3248-3259.

13. Tropres I, Grimault S, Vaeth A, et al. Vessel size imaging. Magn Reson Med 2001;45:397-408.

14. Tropres I, Lamalle L, Peoc'h M, et al. In vivo assessment of tumoral angiogenesis. Magn Reson Med 2004;51:533-541.

15. Serduc R, Brauer-Krisch E, Bouchet A, et al. First trial of spatial and temporal fractionations of the delivered dose using synchrotron microbeam radiation therapy. $J$ Synchrotron Radiat 2009; 16:587-590.

16. Laissue JA, Geiser G, Spanne PO, et al. Neuropathology of ablation of rat gliosarcomas and contiguous brain tissues using a microplanar beam of synchrotronwiggler-generated X rays. Int J Cancer 1998;78:654-660.

17. Serduc R, Bouchet A, Bräuer-Krisch E, et al. Microbeam radiation therapy parameters optimization for rat brain tumors palliation. Influence of the microbeam width at constant valley dose. Submited Phys Med Biol 2009. 
18. Maniotis AJ, Folberg R, Hess A, et al. Vascular channel formation by human melanoma cells in vivo and in vitro: Vasculogenic mimicry. Amer J Pathol 1999;155:739-752.

19. Kim JH, Khil MS, Kolozsvary A, et al. Fractionated radiosurgery for 9L gliosarcoma in the rat brain. Int J Radiat Oncol Biol Phys 1999;45:1035-1040.

20. Kim JH, Chung YG, Kim CY, et al. Upregulation of VEGF and FGF2 in normal rat brain after experimental intraoperative radiation therapy. $J$ Korean Med Sci 2004;19:879-886.

21. Verhoeff JJ, Stalpers LJ, Claes A, et al. Tumour control by whole brain irradiation of anti-VEGF-treated mice bearing intracerebral glioma. Eur J Cancer 2009.

22. Piette C, Munaut C, Foidart JM, et al. Treating gliomas with glucocorticoids: from bedside to bench. Acta Neuropathol 2006;112:651-664.

23. Andratschke NH, Nieder C, Price RE, et al. Potential role of growth factors in diminishing radiation therapy neural tissue injury. Semin Oncol 2005;32:S67-70.

24. Serduc R, Brauer-Krisch E, Siegbahn EA, et al. High-precision radiosurgical dose delivery by interlaced microbeam arrays of high-flux low-energy synchrotron X-rays. PLoS One 2010;5:e9028. 
Figure 1: MRI follow-up, survival curves and lesion size evolution. $T_{2}$-weighted $M R$ images of a non-irradiated (Aa-c) and of a MRT-treated (Ad-h) 9LGS bearing rats at different delays after implantation. B- Individual values (plots) and means (lines) of tumor volumes measured on $\mathrm{T}_{2}$-weighted images at different delays after tumor inoculation for the untreated and MRT-treated groups. Circle and triangle symbols represent the untreated and MRTtreated groups, respectively. C- Kaplan Meier curves showing the survival of 9LGS tumor bearing rats. Untreated controls are represented in grey, while animals treated by MRT are plotted in black. The MRT treatment induced a significant increase in the median survival time of animals (D65 versus D20, log rank test, $\mathrm{p}=0.0003$ ).

Figure 2: Temporal immunohistological follow-up of untreated and MRT treated tumors. HE (a-h) staining and Ki67 ((i-p) red, DAPI counterstained nuclei, blue) immunolabeling observed in irradiated $(\mathrm{MRT}+)$ and non-irradiated tumors (MRT -) at different delays after implantation. Scale bars: $200 \mu \mathrm{m}$.

Figure 3: MRI vessel parameter measurements. BVf (a), VSI (b) and vessel permeability (c) values measured by MRI in the tumor, in the contralateral and ispsilateral hemispheres at different times after tumor inoculation.

Figure 4: Temporal immunohistological follow-up of unirradiated and MRT-irradiated normal and tumoral brain vessels. Type-IV collagen (red) and RECA-1 (green) immunolabeling observed in tumors, in the contralateral (Contra) and ipsilateral (Ipsi) hemispheres for irradiated (MRT+) and control rats (MRT-) at different delays after implantation. Scale bar: $200 \mu \mathrm{m}$. Numbers on each image correspond to the level of co- 
expression of Type-IV collagen and RECA estimated as described in the Supplementary material.

Figure 5: Confocal images of unirradiated and MRT-irradiated 9LGS blood vessels. Type-IV collagen (red) and RECA-1 (green) immunolabeling observed in irradiated (MRT+) and untreated control tumors (MRT-) at D12 and 18 after implantation. Scale bar: $70 \mu \mathrm{m}$.

Figure 6: VEGF expression evolution. Western blot for VEGF in the contralateral (Contra) and ipsilateral (Ispi) hemispheres and tumors of irradiated (unidirectional (Contra) or crossfired (Ipsi and tumor) irradiations (+)) and control rats (-) at different times after implantation. The VEGF/GADPH ratio is given under each blot for each delay and plotted in the three histograms represented on the right. 

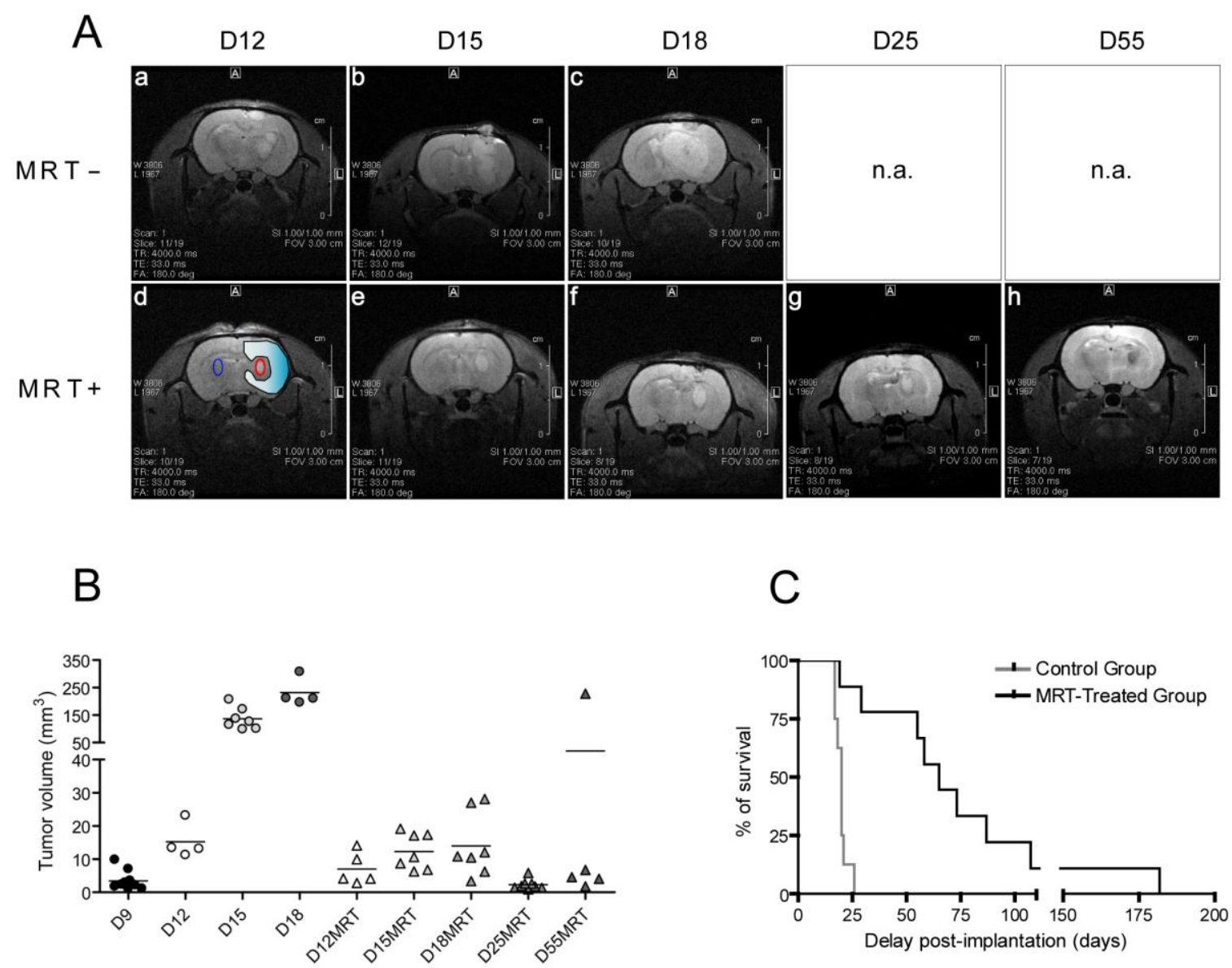

Figure 1: MRI follow-up, survival curves and lesion size evolution. $T_{2}$-weighted $M R$ images of a non-irradiated (Aa-c) and of a MRT-treated (Ad-h) 9LGS bearing rats at different delays after implantation. B- Individual values (plots) and means (lines) of tumor volumes measured on $\mathrm{T}_{2}$-weighted images at different delays after tumor inoculation for the untreated and MRT-treated groups. Circle and triangle symbols represent the untreated and MRTtreated groups, respectively. C- Kaplan Meier curves showing the survival of 9LGS tumor bearing rats. Untreated controls are represented in grey, while animals treated by MRT are plotted in black. The MRT treatment induced a significant increase in the median survival time of animals (D65 versus D20, log rank test, $\mathrm{p}=0.0003$ ). 


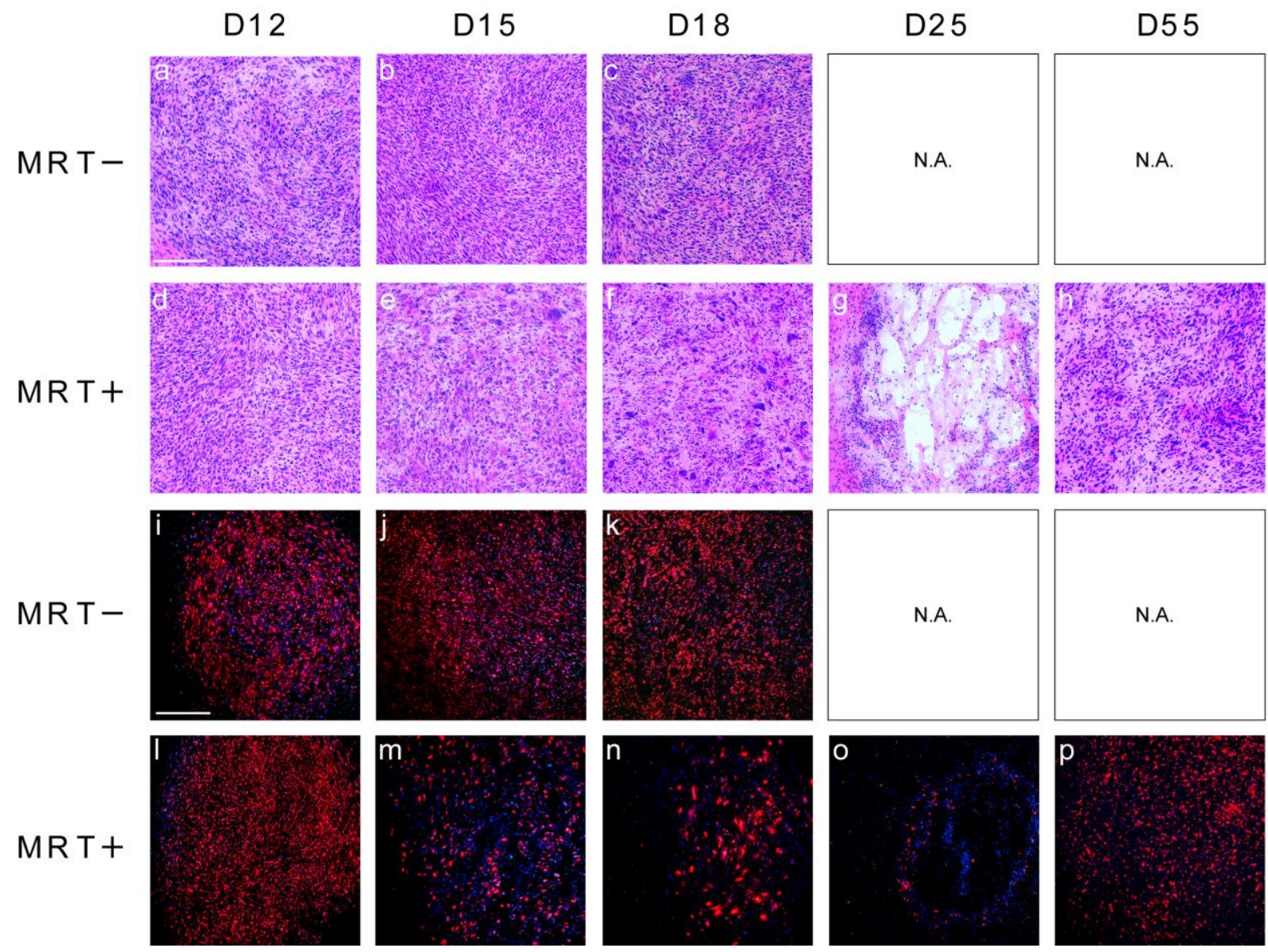

Figure 2: Temporal immunohistological follow-up of untreated and MRT treated tumors. HE (a-h) staining and Ki67 ((i-p) red, DAPI counterstained nuclei, blue) immunolabeling observed in irradiated $(\mathrm{MRT}+)$ and non-irradiated tumors (MRT -) at different delays after implantation. Scale bars represent $200 \mu \mathrm{m}$. 

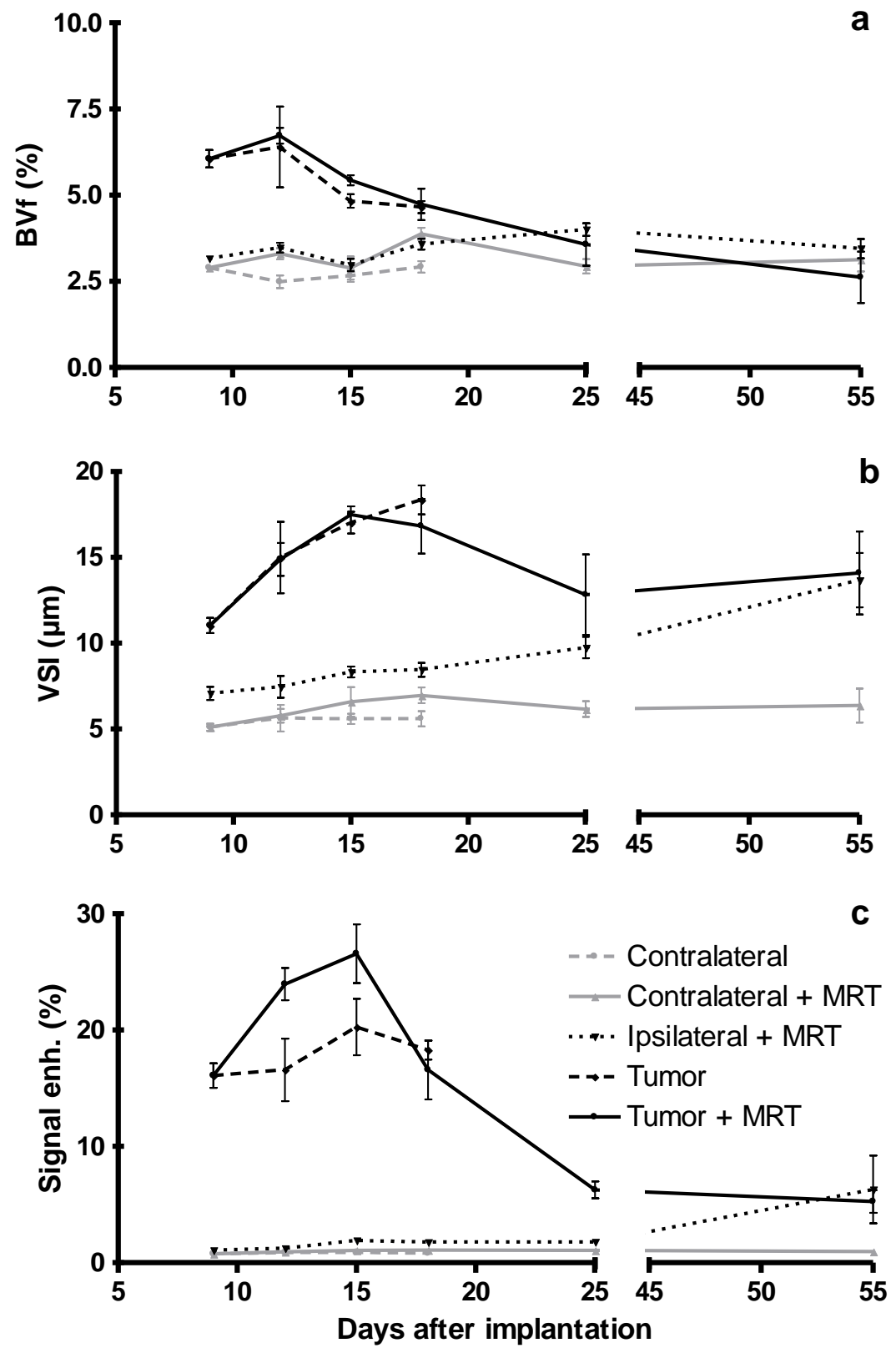

Figure 3: MRI vessel parameter measurements. BVf (a), VSI (b) and vessel permeability (c) values measured by MRI in the tumor, in the contralateral and ispsilateral hemispheres at different times after tumor inoculation (mean $\pm \mathrm{SD}$ ). 


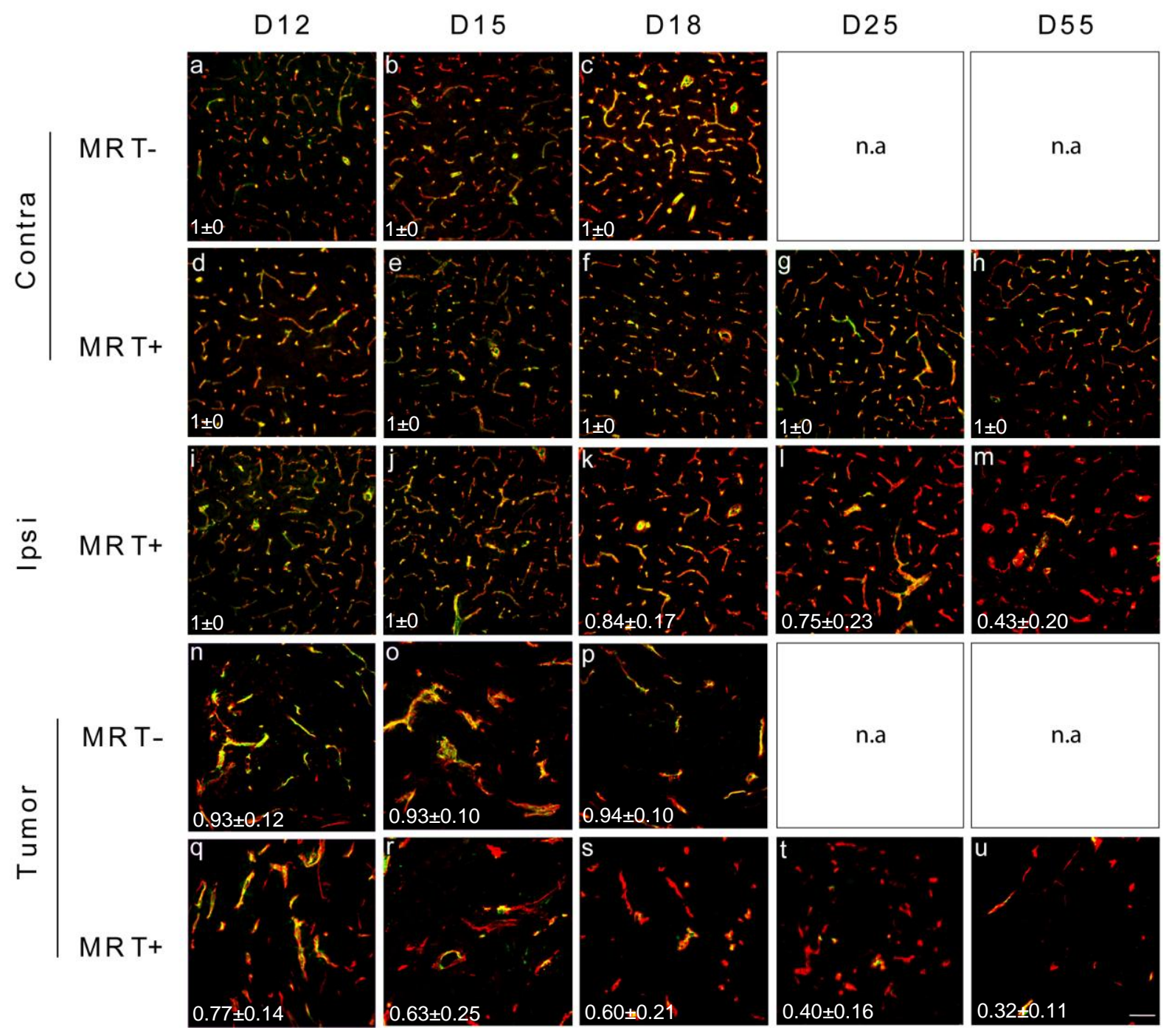

Figure 4: Temporal immunohistological follow-up of unirradiated and MRT-irradiated normal and tumoral brain vessels. Type-IV collagen (red) and RECA-1 (green) immunolabeling observed in tumors, in the contralateral (Contra) and ipsilateral (Ipsi ) hemispheres for irradiated $(\mathrm{MRT}+)$ and control rats (MRT -) at different delays after implantation. Scale bar represents $200 \mu \mathrm{m}$. Numbers on each image correspond to the level of co-expression of Type-IV collagen and RECA estimated as described in the Supplementary material. 

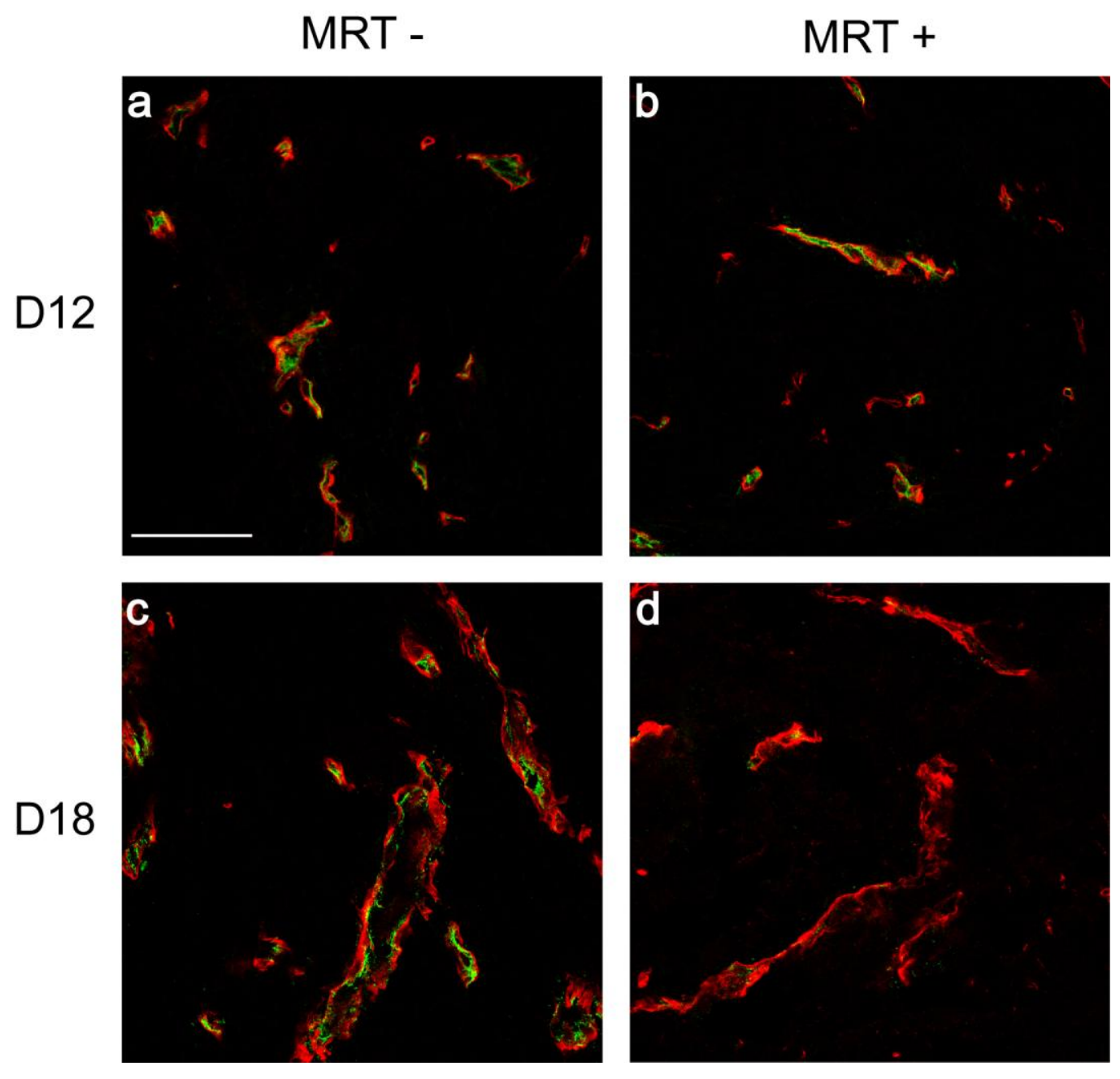

Figure 5: Confocal images of unirradiated and MRT-irradiated 9LGS tumoral blood vessels. Type-IV collagen (red) and RECA-1 (green) immunolabeling observed in irradiated $(\mathrm{MRT}+)$ and untreated control tumors (MRT -) at D12 and 18 after implantation. Irradiated tumor vessels do not exhibit RECA-1 labelling at T8 after treatment. Scale bar represents 70 $\mu \mathrm{m}$. 

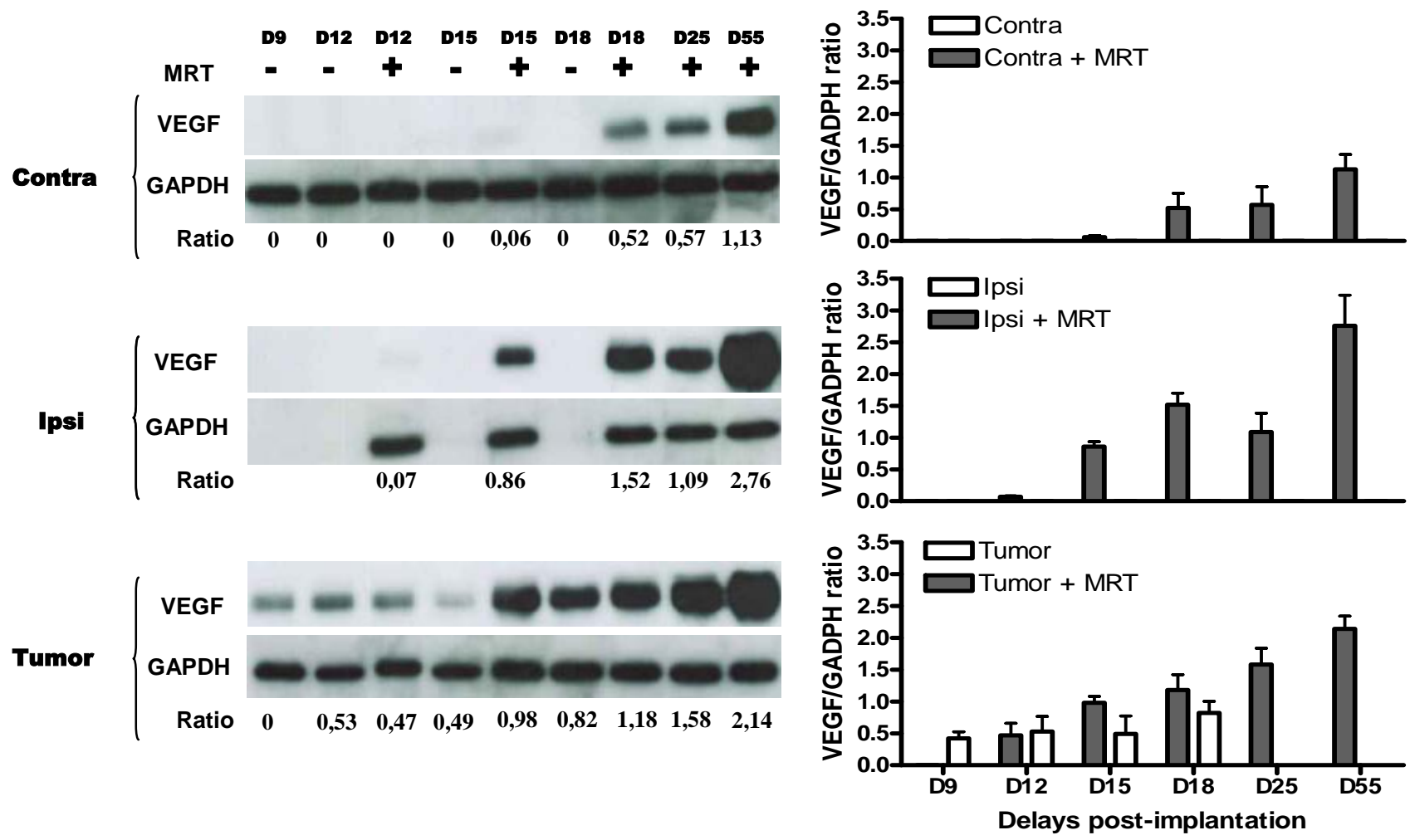

Figure 6: VEGF expression evolution. Western blot for VEGF in the contralateral (Contra) and ipsilateral (Ispi) hemispheres and tumors of irradiated (unidirectional (Contra) or crossfired (Ipsi and tumor) irradiations (+)) and control rats (-) at different times after implantation. The VEGF/GADPH ratio is given under each blot for each delay and plotted in the three histograms represented on the right. 